\title{
The role of experience and advanced training on performance in a motorcycle simulator
}

\author{
David Crundall ${ }^{1}$, Alex W. Stedmon ${ }^{2}$, \\ Elizabeth Crundall ${ }^{3}$, and Rossukorn Saikayasit ${ }^{3}$ \\ ${ }^{1}$ Division of Psychology, School of Social Sciences, \\ Nottingham Trent University \\ ${ }^{2}$ Human Systems Integration Group, Faculty of Engineering and Computing, Coventry \\ University \\ ${ }^{3}$ Human Factors Research Group, Faculty of Engineering, \\ University of Nottingham, UK
}

Keywords: motorcycle; riding behaviour; experience; advanced training

This paper was published in Accident Analysis and Prevention 73 (2014) 81-90.

All copyright rests with this journal

Address for Correspondence:

Prof. David Crundall

Division of Psychology

School of Social Sciences

Nottingham Trent University

Burton Street

Nottingham, NG1 4BU.

+44(0) 1158482358

david.crundall@ntu.ac.uk 


\begin{abstract}
Motorcyclists are over-represented in collision statistics. While many collisions may be the direct fault of another road user, a considerable number of fatalities and injuries are due to the actions of the rider. While increased riding experience may improve skills, advanced training courses may be required to evoke the safest riding behaviours. The current research assessed the impact of experience and advanced training on rider behaviour using a motorcycle simulator. Novice riders, experienced riders and riders with advanced training traversed a virtual world through varying speed limits and roadways of different curvature. Speed and lane position were monitored. In a comparison of $60 \mathrm{mph}$ and $40 \mathrm{mph}$ zones, advanced riders rode more slowly in the $40 \mathrm{mph}$ zones, and had greater variation in lane position than the other two groups. In the $60 \mathrm{mph}$ zones, both advanced and experienced riders had greater lane variation than novices. Across the whole ride, novices tended to position themselves closer to the kerb. In a second analysis across four classifications of curvature (straight, slight, medium, tight) advanced and experienced riders varied their lateral position more so than novices, though advanced riders had greater variation in lane position than even experienced riders in some conditions. The results suggest that experience and advanced training lead to changes in behaviour compared to novice riders which can be interpreted as having a potentially positive impact on road safety.
\end{abstract}




\section{INTRODUCTION}

For the last decade, motorcycles on UK roads have accounted for only $1 \%$ of the 300 billion vehicle miles travelled in any single year (DfT, 2012a), yet in 2011 they accounted for $18.5 \%$ of the 1901 fatalities that occurred, and $21.5 \%$ of collisions that resulted in a Killed or Seriously Injured (KSI) outcome (DfT, 2012b). Many such fatalities and serious injuries are likely to arise from the vulnerable nature of the motorcyclist: without the relative protection afforded to car drivers by the vehicle shell, ostensibly innocuous collisions at relatively slow speeds can still result in injury or death. However motorcycles are also involved in $9.5 \%$ of all recorded collisions regardless of severity (DfT, 2012b) suggesting that, not only are riders more likely to be severely injured compared to other road users following a collision, but they are also more likely to be involved in a road collision. While many of these crashes may actually be due to the behaviour of other road users (Clarke, Ward, Bartle and Truman, 2007; Crundall, Crundall, Clarke and Shahar, 2012), a sizeable number of motorcycle crashes are due to rider error. These may be due to losing control of the motorcycle on a bend (Clarke, et al., 2007), or an inappropriate choice of speed or manoeuvre (Carroll and Waller, 1980; Lynham et al., 2001; Mannering and Grodsky, 1995).

A number of recent studies have attempted to understand and ameliorate the heightened crash risk of motorcyclists (Hosking, Liu and Bayly, 2010; Liu, Hosking and Lenné, 2009; Shahar, Poulter, Clarke and Crundall, 2010; Vidotto, Bastianelli, Spoto and Sergeys, 2011), often focusing on trying to identify what advantages motorcycling experience or specific training might convey. It is generally hoped that studies of this sort might lead to successful interventions, by either distilling the most pertinent benefits of experience or by identifying the most successful elements of advanced training. It is the aim of the current paper to contribute to this literature by identifying measures of motorcycling behaviour that improve with increased experience and/or advanced training. 


\section{The role of experience and training on crash risk}

It is reasonable to expect that both experience with a particular vehicle and advanced training in use of that vehicle should reduce the crash liability of individuals. For instance, in regard to car driving many researchers point to the fact that newly-qualified drivers (usually classified as being within 12 months of passing their driving test) tend to be over-represented in traffic collision statistics (e.g. Braitman, Kirley, McCartt and Chaudhary, 2008; Maycock, Lockwood, and Lester, 1991; Underwood, 2007). During this first year of driving, novices consolidate and build upon the driving skills and strategies they have been taught, tuning them to a range of situations that are likely to extend beyond the rarefied events that one might encounter during formal tuition (Groeger and Banks, 2007). Increases in driving experience have been linked with improved situation awareness (e.g. Jackson, Chapman, and Crundall, 2009); better vehicle handling skills (e.g. Quenault and Parker, 1973), more appropriate visual search strategies (e.g. Crundall and Underwood, 1998; Underwood, Chapman, Brocklehurst, Underwood and Crundall, 2003), attention switching (e.g. Arthur, Strong and Williamson, 1994; Crundall 2009), hazard perception skill (e.g. Horswill and McKenna, 2004), and greater peripheral vision (e.g. Crundall, Underwood and Chapman, 1999, 2002).

Similarly, studies of advanced training techniques have noted improvements in car driver behaviour. For instance Crundall, Andrews, van Loon and Chapman, (2010) found that drivers who had been given a course in commentary driving devised by the police, responded more appropriately to hazards in a driving simulator. Certain advanced training courses have also been related to reductions in crashes (Divey, 1991; Hoinville, Berthould and Mackie, 1972), while other courses or interventions have demonstrated improvements in eye 
movements to hazardous areas of the scene and an increase of anticipatory scanning (Chapman, Underwood and Roberts, 2002; Pollatsek, Narayanaan, Pradhan, and Fisher, 2006); more sensitive risk perception (Rosenbloom, Shahar, Elharar and Danino, 2008), and decreased latencies on hazard perception tests (e.g. Horswill, Kemala, Wetton, Scialfa and Pachana, 2010).

On the basis of these studies one might be forgiven for expecting something akin to a linear improvement with increased experience, with an additive benefit for advanced training. However the literature does not universally endorse experience and advanced training as beneficial. For instance, there is evidence that enduring expectations may be built up with years of driving experience that may have a negative impact on one's ability to respond to highly infrequent hazards. This has been noted in drivers' tendencies to pull out from side roads into the path of oncoming motorcycles, where the driver often reports having looked but failed to see the motorcycle. Crundall et al., (2012) have found oculomotor evidence that suggests experienced car drivers may be more prone to look-but-fail-to-see errors with motorcycles at T-junctions (compared to novice drivers and experienced drivers who also ride motorcycles). We argue that this is due to reduced expectancies for motorcycles that are built up over years on the basis of limited exposure. Simply put, the more often that one pulls out of a junction without there being an approaching motorcycle, the less likely one is to expect an approaching motorcycle in similar future situations. This effectively reduces the cognitive conspicuity of approaching riders. Similar negative expectancy effects have been reported in relation to bicycles (Summala, Pasanen, Räsänen and Sievänen, 1996) and in one documented case of a UK driver using visual strategies based on left-hand driving, on arrival in France (where cars drive on the right; van Elsland and Faucher-Alberton, 1996), though both of these examples are failures to look, rather than true look-but-fail-to-see errors. All of 
these studies demonstrate how enduring expectations built up over time can lead to highly experienced drivers being susceptible to extremely infrequent but dangerous events.

Other studies have suggested that advanced training might also increase specific crash risk. Gregersen (1996) found that training in vehicle handling during a skid increased confidence more than it increased skill, and a number of other Norwegian studies have related such increased confidence to a rise in collisions (cf. Katila et al., 2004). These results have been linked to theories that suggest drivers adapt behaviour towards an optimal level of risk, potentially increasing risky behaviour as their confidence rises (e.g. Risk Homeostasis: Wilde, 1982, 1988; and Risk Allostasis, Fuller et al., 2008).

In perhaps the most pertinent study, Duncan, Williams and Brown (1991) compared novice drivers' on-road performance with that of a group of experienced drivers and a group of advanced drivers who had undertaken an advanced driver training course with the Institute of Advanced Motorists (IAM): a UK charity focussed on improving road safety. They identified a number of tasks where novices appeared to perform better than the experienced drivers, including mirror checks, appropriate visual checks at roundabouts, and appropriate braking on approach to a junction. In some cases the novice and advanced drivers performed similarly, while the experienced drivers did not perform so well. They argued that the experienced drivers tended to perform poorly on those tasks where negative feedback was rare: while a poor gear change may give immediate feedback in terms of noise and judder, a failure to check a mirror will only give negative feedback if a hazard arises (causing a collision, near collision, or at least the audible response of another irate road user). As hazards are less common than the immediate feedback of poor vehicle control, and the mapping is less consistent between action and outcome, Duncan et al., argued that certain skills may not improve or may even decline for experienced drivers. Novice and advanced drivers however benefit from recent explicit feedback given by instructors that maintains 
certain behaviours (e.g. blind spot checks) and allows improvement in skills that might otherwise languish.

In the Duncan et al. study (1991) the car drivers with advanced training did not seem negatively affected by an increase in confidence, perhaps because the training contained a significant focus on awareness of hazards and management of risk (cf. Katila, et al., 2004). Indeed the advanced training ostensibly allowed these drivers to overcome any negative problems of accrued experienced, suggesting that this type of continuous training might not only impart new skills, but might also remediate some bad habits that have developed over the years. Does this translate to motorcyclists? Certainly the current advanced motorcycle training offered by IAM claims to focus on both progression and safety, trying to balance improvements in skill with improvements in risk management. In recently published data we have noted advanced trained riders to have faster response times to hazards in a motorcyclespecific hazard perception test than merely-experienced riders, with novice riders falling nonsignificantly in-between (Crundall, van Loon, Stedmon and Crundall, 2013). The same group of experienced riders were also found to take a more pronounced racing line on a particular S-bend than advanced and novice riders while on a motorcycle simulator (Crundall, Crundall and Stedmon, 2012). The advanced riders took a line that favoured visibility through the curve rather than the 'racing line' which would have taken them closer to the apex of the curve, suggesting that their training had not evoked the sort of risky behaviour that might be expected from an unwarranted increase in confidence. This however was a very specific situation (an S-bend with poor visibility) and it remains to be seen whether these effects of experience and advanced training generalise to more generic situations.

The current study aimed to address the question of whether experience and training are beneficial in regard to two safety-relevant measures: speed maintenance across a varied range of road types (within 40 and $60 \mathrm{mph}$ zones), and bend navigation across a range of 
bends of varying curvature. Following from Duncan et al's (1991) study of car drivers, we anticipated that mere motorcycling experience in itself might not be beneficial on all measures, though advanced training should show a more consistent advantage. Specifically we predict that safety-related measures of speed, braking and lane position may actually be more prevalent in novice and advanced riders, than in riders who merely have experience to support the development and maintenance of skills.

\section{METHOD}

\section{Participants}

Sixty-three motorcycle riders were recruited from the general public to take part, two of whom withdrew part-way through the study due to symptoms consistent with simulator sickness. The 61 remaining participants were classified as either novices, experienced riders or advanced riders. Novice riders $(\mathrm{N}=20 ; 16$ males $)$ were defined as individuals who had completed Compulsory Basic Training (CBT) and were in preparation for the UK Driving Standards Agency motorcycle test, or had passed the standard test within the previous 12 months. Experienced riders $(\mathrm{N}=21 ; 20$ males) were defined as motorcyclists with more than 3 years of experience but who had not undergone any further training. Advanced riders $(\mathrm{N}=$ 20; 18 males) had undertaken an advanced rider training course provided by the IAM not more than three years prior to the study and also had at least 3 years of riding experience. The training course focuses on increasing riders' awareness of hazards and their ability to deal with them, at the same time as improving skill. A detailed breakdown of age and experience can be seen in Table 1. Participants were recruited through local and national advertisements via rider training schools, local motorcycle meetings, and through an on-line newsletter distributed by the IAM. 
<TABLE 1 HERE>

Design

A mixed design was adopted with the different rider groups forming the three levels of the between-subjects factor (novice, experienced, advanced). Two main analyses utilised different within-subject factors. The first analysis compared rider behaviour across $60 \mathrm{mph}$ and $40 \mathrm{mph}$ speed zones. The second set of analyses divided the whole route according to curvature. As by far the majority of curves occurred within the $60 \mathrm{mph}$ zones, the curve analyses were restricted to road segments with a speed limit of $60 \mathrm{mph}$. All $60 \mathrm{mph}$ roads were therefore divided into 4 classifications: straight roads, slight curves with a curvature of 0.001 or less (the reciprocal of the radius), medium curves with curvature up to 0.002 , and tight curves (none of which had curvatures greater than 0.003). Slight curves tended to be 'spirals': portions of road with a very slight curvature that prepare one for entering a bend of greater curvature. Medium and tight curves were full bends in the road.

Several measures were recorded, including average speed and the average time spent above the speed limit, average braking duration, the average number of braking episodes, the average maximum braking effort, the average lateral position of the motorcycle (its position on the road relative to its lane), and the average variance in lateral position (how much the motorcycle changed position in the lane).

In addition to participants' riding behaviour, responses to the Motorcycle Rider Behaviour Questionnaire were also collected. This is a 43 item questionnaire that is reported to produce a five-factor structure, including traffic errors, speed violations, engagement in stunts, use of safety equipment, and control errors (Elliot et al., 2007). 


\section{Apparatus and Stimuli}

Participants rode a virtual route on MotorcycleSim, a motorcycle simulator which employs 'STISIM-Drive v.2' software. The hardware comprises a Triumph Daytona 675 mounted on two pairs of pneumatic actuators. The current study used the simulator in static mode (as opposed to dynamic mode where the rider can physically lean the motorcycle into curves). In the static mode, both pairs of pneumatic actuators were pressurised (at approximately 10bar) and the motorcycle did not lean. This static pressure provided a high degree of stability, though there was enough freedom of lateral movement to give the illusion of instability, which encouraged riders to actively maintain their balance as they would in a real riding situation. A floor-mounted projector presented the virtual environment on a screen measuring $285 \mathrm{~cm} \times 228 \mathrm{~cm}$ with a resolution of $1280 \times 1024$ at a distance of $190 \mathrm{~cm}$ in front of the rider. While riding, participants either wore their own helmet or an open face Arai helmet that was provided for them.

A bespoke scenario was modeled in the 'STISIM-Drive v.2' simulation software that was configured as a motorcycle model. While the motorcycle hardware did not lean or tilt in this current study, the simulated world did tilt to give the visual impression of leaning into a corner. The route comprised two laps of a 9.6 mile circuit. The circuit contained both urban/suburban and rural roads with posted speed limits of $40 \mathrm{mph}$ and $60 \mathrm{mph}$, respectively. The route contained bends, traffic, pedestrians, junctions, traffic lights and road signs. All signs, road markings, and priorities represented the UK road network (with riders travelling on the left of the road), and a complete ride lasted approximately 25 minutes. On the second lap of the circuit riders encountered four pre-defined hazards, two in the $60 \mathrm{mph}$ zone and two in the $40 \mathrm{mph}$ zone. Specific responses to these pre-defined hazards are not considered further in this paper, but are discussed elsewhere (Stedmon, Crundall, Crundall, Saikayasit, 
van Loon, Irune, Ward and Greg, 2011; Crundall, Stedmon, Saikayasit and Crundall, 2013; Crundall, Crundall, and Stedmon, 2012).

\section{Procedure}

Each testing session lasted approximately 70 minutes and participants were paid a $£ 12$ inconvenience allowance. The session began with a general introduction and familiarisation of the simulator controls (brakes, throttle, gears etc.). Prior to sitting on the simulator participants completed a simulator sickness questionnaire (entitled a 'rider comfort check') to provide baseline information. They then underwent two practice sessions, the first without other traffic and the second with a number of slow moving vehicles to promote overtaking manoeuvres. A second simulator sickness check was then administered before riders began the full virtual route comprising two laps, totalling 19 miles. If a participant crashed, the simulator placed the motorcycle at the side of the road at the point of collision and they were allowed to continue when they were ready. At the end of the simulator session a final simulator sickness check was completed, followed by the Motorcycle Rider Behaviour Questionnaire (MRBQ). Other tests were subsequently administered including a workload measure (NASA-TLX), a locus of control test, and a motorcycle hazard perception test (see Crundall, van Loon, Stedmon and Crundall, 2013; Stedmon, et al, 2011), though these additional tests will not be discussed further in this paper.

\section{RESULTS}

The results section reports data from the Motorcycle Riding Behaviour Questionnaire, followed by measures of speed and lane position for different speed zones, and a comparison of straight and curved road sections. In addition, the number of crashes was recorded. While crash frequency produced insufficient data for analysis (hence the need for surrogate measures such as speed and lane position), they are reported here for completeness. There 
were a number of pre-specified hazards placed within the route which some riders collided with, while other crashes occurred in the absence of designed hazards, primarily on bends in the $60 \mathrm{mph}$ zone. The frequency of these crashes is detailed in Table 2. Further details on collisions and the specific riding behaviour that preceded crashes with pre-specified hazards can be found elsewhere (Crundall, Stedmon, Saikayasit, and Crundall, 2013; Crundall, Crundall and Stedmon, 2012; Stedmon, et al., 2011).

\section{$<$ TABLE 2 HERE >}

Prior to the analysis of the simulator data, the scores from the Motorcycle Riding Behaviour Questionnaire were collated. Cronbach's alpha was calculated for the five factors that were identified by Elliot et al. (2007). The alphas, and the mean scores across groups, are presented in Table 3. Only three of these factors reached the 0.7 criterion threshold for acceptance, therefore only traffic errors, speed violations and stunts were analysed further. Scores on each of these three factors were compared across the three rider groups with a series of 1 x 3 Analyses of Variance (ANOVA) tests.

\section{<TABLE 3 HERE>}

Analysis of both the traffic errors factor and the speed violations factor revealed a group effect $(\mathrm{F}(2,58)=6.2, \mathrm{MSe}=0.1, \mathrm{p}<0.005$, and $\mathrm{F}(2,58)=8.4, \mathrm{MSe}=0.4, \mathrm{p}<0.001$, respectively). In both cases, Tukey HSD tests identified the advanced riders to have lower ratings than both the experienced riders and the novice riders (all at $\mathrm{p}<0.05$ or lower). There was no differentiation between rider groups in relation to the factor of Stunts, however, as can be seen in Table 3, all participants' ratings were extremely low on this factor. 


\section{Rider behaviour in 60 and 40 mph zones}

The following section details the analysis of all riding as a function of which speed limit zone the rider was in, while the subsequent section will detail the comparisons of straight roads with the three classifications of road curvature.

The average speed of the three rider groups across the two different speed limit zones was subjected to a $2 \times 3$ Analysis of Variance (ANOVA). While it was unsurprising to find that riders had a higher speed in the $60 \mathrm{mph}$ zone compared to the $40 \mathrm{mph}$ zone (means $=55$ mph vs. 37 mph respectively; $F(1,58)=1223, \mathrm{MSe}=8.2, \mathrm{p}<0.001)$, it was more interesting to note an interaction between the speed limit zone and rider group $(\mathrm{F}(2,58)=3.6, \mathrm{MSe}=8.2$, $\mathrm{p}<0.05$ ). As can be seen in Figure 1 (left panel) all riders tended to have similar speeds in the $60 \mathrm{mph}$ zone. It was only in the $40 \mathrm{mph}$ zone that a difference between groups was noted, with the advanced riders $($ mean $=35.0 \mathrm{mph})$ travelling more slowly than the novice $($ mean $=$ $37.6 \mathrm{mph}$ ) and experienced riders (mean $=37.1 \mathrm{mph}$; Tukey HSD, $\mathrm{p}<0.05)$.

\section{$<$ FIGURE 1 HERE >}

While average speed measures were within the posted speed limits for the respective zones, there were still periods of time when riders exceeded these limits, albeit not sufficiently to raise the average speed of their group above the speed limit. The average duration that participants spent above the speed limit in the $60 \mathrm{mph}$ and $40 \mathrm{mph}$ limit zones was therefore subjected to a 2 x 3 ANOVA. An interaction fell short of conventional statistical acceptance $(\mathrm{F}(2,58)=2.6, \mathrm{MSe}=149, \mathrm{p}=0.08)$ though it suggests a trend that fits with the average speed data. As can be seen in Figure 1 (right panel) there is a suggestion that the advanced riders exceeded the speed limit the least, while the novices speeded the most, 
but only in the $40 \mathrm{mph}$ zone. This fits with the average speed analysis which suggests that the advanced riders appeared to be particularly cautious regarding their speed when travelling in the 40 mph zones.

The simulator also recorded the lateral position of the motorcycle in the lane. The width of the lane was 12 feet. The simulator attributes the centre line of the road (the dividing line between the two opposing lanes) with a lateral position score of zero. Riders who remain in their lane vary in lateral position between zero and -12 feet, with greater negative numbers reflecting a lateral position that is further away from the centre line and closer to the kerbside, while positive numbers between zero and +12 feet represent excursions over the centre line into the oncoming traffic lane. The average lateral positions for the three groups of riders were compared with a 2 x 3 ANOVA. A main effect of speed limit revealed that all riders tended to position themselves further from the centre line in $60 \mathrm{mph}$ zones compared to 40 mph zones (means $=-5.2$ feet vs. -4.1 feet $F(1,58)=482, \mathrm{MSe}=0.08, \mathrm{p}<0.001)$. The average position in the $60 \mathrm{mph}$ zone was actually closer to the centre of the lane however. More interestingly, there was also a main effect of rider group $(\mathrm{F}(2,58)=18.0, \mathrm{MSe}=0.67, \mathrm{p}$ $<0.001)$. The data were further subjected to a series of post hoc Tukey HSD tests which showed all rider groups to differ from each other $(\mathrm{p}<0.05)$. Figure 2 (left panel) shows that novices were the furthest to the left (that is, further away from the centre line in the UK road system) while advanced riders were closest to the centre line (means $=-5.2$ feet vs. -4.1 feet, respectively).

In addition to the average lateral position of the riders in the lane, we were also concerned with the variance of their lateral position. This measure refers to how much they changed their lateral position while riding. When recording car driver behaviour in a simulator, an increase in the variance or standard deviation of lane position is often viewed negatively and is linked with increased distraction, drug use, fatigue and alcohol consumption 
(e.g. Verster, Taillard, Sagaspe, Olivier, and Philip, 2011). In regard to motorcycling however we predicted that increased manoeuverability within lanes might be considered a positive attribute: while car drivers cannot vary their lateral position much within a lane without risking a lane excursion, motorcyclists have much more lateral space to use strategically within their lane. This strategic use of lane position could include using the 'racing line' to navigate corners (this is the path through a curve which offers optimum progression, requiring a rider to move from the outside of the curve to the inside at the point of passing the apex, before moving back towards the outside of the curve), choosing a position to maximise visibility, or even engaging in 'lateral weaving' - a technique recommended by the UK Motorcycle Action Group as a possible method for disrupting motion camouflage when approaching side roads with vehicles waiting to pull out (MAG UK, 2006).

A $2 \times 3$ ANOVA on the variance of lateral position showed greater variance in 60 mph speed zones compared to $40 \mathrm{mph}$ speed zones $(\mathrm{F}(1,58)=149, \mathrm{MSe}=3.3, \mathrm{p}<0.001)$, which was most probably caused by the majority of significant curves occurring in the 60 mph zone, which in turn are likely to have encouraged riders to alter their position. Of greater interest was the effect of rider group $(\mathrm{F}(2,58)=9.2, \mathrm{MSe}=5.4, \mathrm{p}<0.001)$ and an interaction between speed limit zone and rider group $(\mathrm{F}(2,58)=3.8, \mathrm{MSe}=3.3, \mathrm{p}<0.05)$. Post hoc tests revealed the advanced riders to have a greater overall variance in lateral position (mean $=6.1$ feet $)$ than the novices $($ mean $=3.9$ feet, $\mathrm{p}<0.001)$, though the experienced riders $($ mean $=5.1$ feet) fell in-between, failing to differ significantly from either the advanced group or the novices. The reason for this becomes apparent when viewing the interaction (Figure 2, right panel). The graph clearly shows that the experienced riders behave more like the advanced group in the $60 \mathrm{mph}$ zones, but more like the novices in the $40 \mathrm{mph}$ zones. Again this argues that advanced training is evoking specific behaviours in these riders within the $40 \mathrm{mph}$ zones which may be providing a safety benefit. 


\section{$<$ FIGURE 2 HERE >}

Several measures of braking were also recorded, but the only measure to show an effect of rider group was the average maximum braking effort. This was calculated by recording the maximum pressure applied to the front and rear brakes (with a 25:75 weighting) during any one braking episode, and then averaging them for each rider within the $60 \mathrm{mph}$ and $40 \mathrm{mph}$ speed limit zones. They are reported as percentages of the maximum braking that could be applied. The 2 × 3 ANOVA on these data revealed an interaction $(\mathrm{F}(2,58)=6.7$, MSe $=89.9, \mathrm{p}<0.005)$ which is clearly identifiable in Figure 3: while all groups have similar maximum braking scores in the $60 \mathrm{mph}$ zone, the advanced riders have a much greater maximum braking effort in the $40 \mathrm{mph}$. This is again congruent with the suggestion that advanced training has had greater impact on riding behaviour in the $40 \mathrm{mph}$ zone than in the $60 \mathrm{mph}$ zone, though it should be noted that does not necessarily imply safer behaviour in the advanced group.

<FIGURE 3 HERE>

\section{A comparison of rider behaviour across straights and curves}

In addition to the comparison of behavioural measures across the $40 \mathrm{mph}$ and $60 \mathrm{mph}$ zones, a second set of analyses were conducted across varying curvature characteristics of road sections. As the majority of curves tended to occur in the $60 \mathrm{mph}$ zones, all data from $40 \mathrm{mph}$ zones were excluded. The road sections in the $60 \mathrm{mph}$ zones were either classified as straights, slight curves (preparatory 'spirals'), medium curves or tight curves. The first 
analysis compared average speed in a $3 \times 4$ ANOVA across rider group and the four classifications of curvature. Across all riders, speed was the greatest on the straights $($ mean $=$ $59.9 \mathrm{mph}$ ), reducing on the slight curves $($ mean $=49.9 \mathrm{mph}$ ) as riders prepared for tighter curves ahead. Medium curves required no further adjustment in speed (mean $=50.2 \mathrm{mph}$ ), though tight curves tended to provoke a further decrease in speed (mean $=47.4 \mathrm{mph}$ ), as shown by planned contrasts comparing speed on the straight road sections, and the medium and tight curves, to the speed on the slight curves. The rationale for these comparisons was that the slight curves are the transition point between straights and either medium or tight curves. The reduction in speed across these four road types varied across the rider groups however, as noted in the interaction $(\mathrm{F}(6,174)=5.2, \mathrm{MSe}=8.7, \mathrm{p}<0.001)$. Planned interaction contrasts revealed the interaction to lie in two places, between straights and slight curves and between medium curves and slight curves $(\mathrm{F}(2,58)=5.5, \mathrm{MSe}=27.6, \mathrm{p}<0.01$, and $\mathrm{F}(2,58)=3.8, \mathrm{MSe}=2.9, \mathrm{p}<0.05$, respectively). As can be seen in Figure 4 (left panel) all riders reduced their speed from straights to slight curves, but experienced riders reduced their speed to a greater extent than the other two groups. Between slight curves and medium curves the novices and experienced riders maintained their speed, however it appears that the advanced riders had a slight but significant increase in their speed on medium curves.

The absolute lane position was not compared across rider groups for different curves as the data were collapsed across left and right bends. Of greater interest was the variance of lateral position across the four road segments as this measure is more sensitive to whether riders decide to take a racing line through a bend (which would require them to move from one side of the lane to the other, and back again, resulting in a high variance in lateral position). A 3 x 4 ANOVA revealed a main effect of rider group $(\mathrm{F}(2,58)=5.2, \mathrm{MSe}=11.6$, $\mathrm{p}<0.01$ ). Tukey HSD tests confirmed that novices (mean $=6.3$ feet) had much less variance in their lateral position compared to the advanced riders (mean $=9.6$ feet, $\mathrm{p}<0.01$ ), with 
marginal evidence for less variation in lane position compared to experienced riders (mean = 8.8 feet, $\mathrm{p}=0.05)$. Experienced riders and advanced riders did not differ.

This effect was further qualified by an interaction between rider group and curve type $(\mathrm{F}(6,174)=9.2, \mathrm{MSe}=6.1, \mathrm{p}<0.001)$. Planned interaction contrasts, again comparing speed on the straight, medium and tight curves to the speed on the slight curves, revealed effects at every level of geometry when compared to behaviour in slight curves. The results were further investigated by breaking down the analysis into four 1x3 ANOVAs comparing the three groups at each level of road geometry. Group effects were only noted for the straight sections $(\mathrm{F}(2,58)=22.9, \mathrm{MSe}=2.8, \mathrm{p}<0.001)$ and the slight curves $(\mathrm{F}(2,58)=10.2, \mathrm{MSe}=$ 25.6, $\mathrm{p}<0.001)$. Tukey tests suggested that advanced riders had greater variation in lane position than both experience and novice riders on straights $(\mathrm{p}<0.001)$, while the latter two groups did not differ. On slight bends novices had less variation than advanced riders $(\mathrm{p}<$ 0.001), while the experienced riders fell in-between, with marginal evidence for having more variance than novices $(\mathrm{p}=0.06)$ and less variance than advanced riders $(\mathrm{p}=0.07)$. While increased variance in lane position is generally considered to symptomatic of distraction or lack of experience in drivers, these results raise the possibility that lane variance is a positive attribute on straights as well as preparatory curves for motorcyclists.

\section{$<$ FIGURE 4 HERE>}

When measures of braking were subjected to similar 3 x 4 ANOVAs, no rider effect or interaction was noted for braking duration, the number of braking episodes, or the average maximum braking.

\section{DISCUSSION}


The current study aimed to identify the effects of experience and advanced training on several measures taken from a simulated motorcycle ride through a virtual route. The literature is replete with evidence that driving and, to a lesser extent, riding experience correspond to an increase in safety-related skills such as vehicle handling, visual search and hazard perception (e.g. Crundall and Underwood, 1998; Horswill and McKenna, 2004). Various forms of advanced training have also shown beneficial effects (Divey, 1991; Rosenbloom, et al., 2008; Horswill, et al., 2010). It is therefore natural to postulate that all safety-related measures for driving or riding should improve with experience and advanced training. However, we have also noted evidence that those behaviours which rarely receive negative feedback may be less prone to experiential development, and may rely solely on explicit training, often at an advanced level. Unfortunately there is also evidence that certain training approaches may increase crash liability if it fails to inoculate trainees against over-confidence (Gregersen, 1996; Katila et al., 2004).

The measures taken in this study related to speed control and lateral behaviour. While speed and lane maintenance are required every time one rides a motorcycle, it remains possible that negative feedback regarding these behaviours is still relatively rare (such as encountering an infrequent hazard or coming close to losing control on a bend, which then makes riders re-evaluate their own riding style). Thus it was possible that some of the measures would not demonstrate an experiential benefit. We predicted that advanced training should show a more consistent advantage, though the possibility remained of training impacting negatively on riding style, perhaps through risk allostasis or homeostasis (e.g Wilde, 1982, 1988; Fuller et al., 2008).

The initial measures from the Motorcycle Riding Behaviour Questionnaire provided the first evidence for a positive benefit of advanced training over mere experience, with the 
advanced riders reporting fewer traffic errors and speed violations. While these self-report measures may merely reflect the additional motivation of the advanced riders to present a more safety-conscious image than the other two groups, the behavioural data are consistent with more conscientious speed maintenance in the advanced group. Advanced riders rode more slowly in the $40 \mathrm{mph}$ zone, and there was marginal evidence for less frequent speed violations in the $40 \mathrm{mph}$ zone. Novices however appeared the most likely to speed in the 40 mph zone. In regard to the comparison of $40 \mathrm{mph}$ and $60 \mathrm{mph}$ zones, advanced riders also took up a more central position in the road, closer to the centre line, while novices tended to position themselves significantly closer to the kerbside. There are a number of advantages to the central position chosen by the advanced riders: it provides good visibility of potential hazards approaching from either side of the main carriageway, it maximises the distance from parked vehicles and avoids drains, the camber of the road is at a minimum, and it potentially increases the probability of other road users looking at the motorcycle. It is possible that the kerbside positioning seen with the novice riders could be an over-compensation for the potential hazard of oncoming traffic. Alternatively one might postulate that the novice riders, being younger, are more likely to have recently been dependent on cycling as their main mode of transport. Cycling requires a kerbside position in order to allow vehicles to pass, and it is possible that this strategy carries over when one starts to ride a motorcycle, creating a slight kerbside bias. As we did not collect any data on bicycle usage, it is impossible to comment further on this particular post hoc rationalisation.

Despite the advanced riders maintaining a relatively central road position (i.e. to the inside of their lane), they also varied their lane position more than both other groups in the 40 mph zone, and more than the novices in the $60 \mathrm{mph}$ zone. This raises a number of suggestions regarding the behaviour of the advanced riders. First, their increased variance of lane position in the $40 \mathrm{mph}$ zone suggests that this is independent of a desire to adopt a racing 
line, as there were few curves in the $40 \mathrm{mph}$ zones. Instead, the advanced riders must be changing position according to other elements in the scene such as side roads, parked vehicles, oncoming vehicles and pedestrians. Both novice and experienced riders were more likely to maintain their lane position through these zones, appearing relatively insensitive to the vagaries of other simulated road users. This conclusion conflicts with the typical conclusion derived from lane variability in driving, where changes in lane position are often considered to reflect distraction or a processing overload (e.g. Verster, et al, 2011). However there were no obvious demands placed on the advanced riders in the $40 \mathrm{mph}$ zones that were not also present for the other groups. There were few bends, and the only reason to change lane position was in response to the potential hazards in the $40 \mathrm{mph}$ zone such as parked or moving vehicles. Logically, it also makes sense to use a motorcycle's lane position as a flexible safety buffer. Lane variance while driving a car risks lane excursion; a risk which is greatly lowered with the narrow profile of a motorcycle and which maybe outweighed by the benefits of lateral variation.

The overall increase in lane variance from the $40 \mathrm{mph}$ to the $60 \mathrm{mph}$ zones is most likely explained by the curves evoking changes in lane position (either through adoption of the racing line, or positioning in such a way as to increase visibility through bends). Experienced riders changed lane position as much as the advanced riders in the $60 \mathrm{mph}$ zones. The current data do not allow us to identify whether the racing line or visibility was the key concern, though other data suggest that experienced riders' lane variance might be explained by the racing line, while advanced riders' position might be better explained by maximising visibility through bends (Crundall, Crundall and Stedmon, 2012). The fact that advanced riders show increased lane variance in both the $40 \mathrm{mph}$ and $60 \mathrm{mph}$ zones (where only the latter contains a significant number of bends) fits with the suggestion that their lane position varies, at least in part, for safety/visibility issues. As experienced riders only show 
increased variance in the $60 \mathrm{mph}$ zone, it suggests that progression via the racing line is more likely. While the novice riders also showed a willingness to change lane position in the 60 mph zone compared to the $40 \mathrm{mph}$ zone, their variation of lane position was much less than the other two groups. This suggests that novices are either trying to take the racing line but are not yet at a level of experience where they can commit as fully as the other riders, or they perceive the risks of the $60 \mathrm{mph}$ zone to be greater than those in the $40 \mathrm{mph}$ zone.

The braking data follow a similar pattern, showing that advanced riders are particularly responsive in the $40 \mathrm{mph}$ zone compared to the other rider groups. However it should be noted that the braking measure that revealed the interaction was the average maximum braking pressure. This suggests relatively 'hard braking' in response to events and surroundings in the $40 \mathrm{mph}$ zones. These braking episodes might be responsible for the lower speeds noted for advanced riders in the $40 \mathrm{mph}$ zones. Thus, rather than the advanced riders showing a greater degree of caution in their speed maintenance through $40 \mathrm{mph}$ zones, they may actually adopt a faster speed throughout the majority of the $40 \mathrm{mph}$ zones, punctuated by periods of relatively hard-braking in response to perceived threats. As the number of braking episodes did not differ between groups however it is more likely that a single, relatively hardbraking episode reduced speed for the advanced riders compared to the other groups. The most obvious contender for this would be upon entrance to the $40 \mathrm{mph}$ zone. Data from a detailed sub-section analysis also argues against advanced riders braking more harshly in response to perceived hazards (Crundall et al., 2013).

In summary, the results from the comparison of speed zones reveal an interesting pattern, with experience impacting upon some aspects of riding style, while advanced training appears to influence behaviour even further, especially in the $40 \mathrm{mph}$ zone. Novice riders appear happy to travel at relatively high speeds and even appear the most likely to violate the speed limit in the $40 \mathrm{mph}$ zone. Their lane positioning could be described as 'stable', though 
a better description of this might be 'inflexible', possibly representing a failure of their mental model for lane positioning, or unconscious biases derived from over-caution for oncoming traffic or adapting irrelevant models (e.g. those of a cyclist) to the motorcycling experience. Experienced riders showed greater use of the lane in the $60 \mathrm{mph}$ zone, possibly reflecting them seeking out progression opportunities, but they were insensitive to possible hazards in the $40 \mathrm{mph}$ zones that would benefit from changing lane position or braking. The advanced riders appeared most cautious in regard to speed when travelling through the 40 mph zones, though the measure of maximum braking could be interpreted to suggest that their passage through these zones may still contain periods of relatively high speed. At least equally plausible however is that they make a greater speed reduction on entering the lower speed zone (which in itself is an element of the advanced training programme: to maintain speed within a zone but to quickly adjust speed prior to entering a new speed zone). The evidence for both lower self-reported speed violations, and a decreased period of time speeding in the $40 \mathrm{mph}$ zone argues for a positive interpretation of the advanced riders' speed choice. Similarly, the increase in lane variance, especially in the $40 \mathrm{mph}$ zone when there are no progression advantages to changing lateral position, argues that this behaviour is indicative of a safer riding style. This is in contrast to the literature on driving that considers lane deviation to reflect high workload or distraction.

The second analysis of speed and lane position across the four classifications of curvature revealed another complex pattern. All riders slowed on the slight curves (entry spirals) in preparation for either a medium or tight curve ahead. Experienced riders slowed the most for all types of curves, while advanced riders appeared to increase speed fractionally from slight to medium curves. The advanced riders changed lane position most in the slight curves in preparation for the medium and tight curves, therefore perhaps allowing a faster and more efficient speed through the bends. The novice and experienced riders appeared however 
to show a lag in their changing lane position compared to the advanced riders, with greater or at least equal lane variation for medium and tight curves as in the slight curves. This suggests that the novices and, to a lesser extent, the experienced riders were not preparing for the curve as early as the advanced riders. This cannot however be due to the advanced riders moving towards the curve as if to take an early racing line, as at this point in the entry spiral such a move would result in a rider overshooting the curve. Instead this would seem to reflect the advanced rider moving away from the curve (e.g. on a UK left-hand bend this would mean the advanced rider would move towards the centre line of the road), presumably to provide a better entry angle. This would have the dual benefits of improving the racing line while also increasing visibility through the bend. A more detailed analysis of one particular simulated bend (Crundall, Crundall and Stedmon, 2012) supports this, as advanced riders were found to be only 2 feet to the left of the centre line at the start of the curve (as opposed to over 4 feet away from the centre line on average in the current data; see Figure 2, left panel). This suggests that advanced riders must move even further towards the centre line during slight curves in order to promote the best view through the bend, which then affords them an increase in speed through medium bends.

In conclusion most of the measures appear to support the naive hypothesis of increased improvement with experience and advanced training, though the amount of benefit differs according to the circumstances. Experienced riders have developed their abilities to take a racing line in $60 \mathrm{mph}$ zones (potentially at the expense of a safer road position and viewpoint through the bend), though they have not developed the sensitivity to the potential hazards noted in the $40 \mathrm{mph}$ zone. This may reflect our riding sample to a certain extent: when questioned, $100 \%$ of our experienced riders reported using a motorcycle to ride for pleasure, while only $57 \%$ reported using it for commuting. Thus one could imagine that, on the whole, our experienced riders may have covered more miles in the countryside than in 
urban settings. The advanced riders however appear to have benefited in many areas from their advanced training: they prepare for curves sooner and they are more responsive to their surroundings in $40 \mathrm{mph}$ zones. Thus it appears that the particular advanced training that the advanced group undertook has improved their performance without increasing risk taking via risk homeostasis.

There are two important caveats to add however. First, we cannot completely rule out the possibility that advanced riders are more likely to want to present a positive image during the experiment. While this is an obvious problem for self-report measures such as the MRBQ, it is also possible that riders may have affected a safe riding style for the purposes of the study. Many riders who have undertaken the advanced riding course are members of the organisation that conducts the training, and may therefore feel obliged to ride safely when taking part in a study to assess the extent of their skills. However, it must also be noted that a tendency to ride in a socially responsible and desirable manner could have easily affected the other rider groups also, with novices riding according to their recent formal training and the experienced riders riding in a manner that they assume to be safer.

The second caveat is that while the measures recorded by the simulator are objective, the interpretation of these measures in regard to safe and unsafe behaviour is much more subjective. In many studies of driving experience, differences in behaviour between experienced and novice drivers are often deemed to improve safety on the a priori basis that experienced car drivers have fewer collisions than novice drivers. However, as several studies have demonstrated, experience and training do not always have a positive impact on safety (e.g. Duncan et al., 1991; Gregersen, 1996; Katila et al., 2004). Thus one is left with ambiguity as to the relevance of specific group differences to safety, especially when direct measures of safety such as actual or virtual collisions are so few. For instance, the increased braking measure found for the advanced riders in the $40 \mathrm{mph}$ zone could be viewed as an 
example of them responding too late to potential hazards. Likewise, the increased lane variance could be interpreted very differently if one were to maintain the negative connotations used in relation to car drivers. For the former measure we have suggested that advanced riders appear to be safer in $40 \mathrm{mph}$ zones despite the greater maximum braking measure. It could have been that the advanced riders, travelling on average at a slower speed than the other rider groups, were then able to apply the brakes harder because there was less perceived danger of skidding which would be the case if applying hard braking to a fast moving motorcycle. This was based on the evidence from other measures that were less ambiguous (e.g. slower overall speed), and alternative explanations for the braking measure that were not key to immediate safety (e.g. greater braking on entry to the $40 \mathrm{mph}$ zone). In regard to lane variability, we suggest that the narrow profile of a motorcycle allows lane position to become a flexible optimum in response to the ever-changing roadway.

While we have done our best to interpret these data as fairly as possible with input from psychologists, human factors experts and motorcyclists, we invite other researchers to debate which simulator measures should be considered characteristic of safe riding. While the current crash data are insufficient to support the hypothesis that advanced training really does reduce crash liability, the trend in collisions with pre-specified hazards is promising. Less promising are the five collisions where advanced riders lost control on bends. Could these collisions be linked to the advanced riders adopting a faster speed on medium bends, perhaps through confidence outstripping ability? Though the small number of bend-crashes is insufficient to draw such a conclusion, it is highly probably that advanced riders' confidence in riding a real motorcycle outstrips their performance in a simulator (rather than performance on a real motorcycle). The fidelity of any simulation is likely to be more important to experts than novices. For instance, advanced riders might be more likely to actively exploit counter-steering when cornering (turning the handlebars in the opposite 
direction to travel, causing the motorcycle to lean into a bend). This functionality is not available in the current simulator configuration and was commented on by some of the advanced riders. Similarly, the inability to tilt the motorcycle while travelling around corners may have affected the advanced riders more so than the other groups although no formal comments were made to this effect.

Accepting these caveats, the current data support the findings of Duncan et al (1991) where advanced training was found to improve certain performance measures in car driving above and beyond mere experience. The skills that we measured however did not show poorer behaviour in the experienced riders relative to the novices. Following Duncan et al (1991) this suggests that the measures we adopted in this study are all susceptible to modification from feedback gained through experience. We did not find any convincing evidence to suggest that the advanced riders became more risky, though a strict safety perspective would not advocate advanced training that increases speed (as it appears might have happened with our advanced riders on medium bends). Certainly a focus of advanced training on both progression and safety appears oxymoronic. However while a course purely devoted to safety would potentially be more beneficial, it might be difficult to convince some motorcyclists to take part. While the current results suggest that training in a mixture of progression and safety does change behaviour, and many such changes can be interpreted as a positive influence on safety, further research is required to identify the different components of progression and safety training on different measures of riding behaviour.

\section{Acknowledgements}

This work was funded by the Institute of Advanced Motorists (www.iam.org.uk). The funders had no role in study design, data collection and analysis, decision to publish, or preparation of 
the manuscript. Stedmon is an IAM trained motorcyclist. We would like to thank Editha van Loon, Patrick Ward, Alex Irune and Neil Greig for their assistance in this project.

\section{References}

Arthur, Jr., W., Strong, M. H., and Williamson, J., (1994). Validation of a visual attention test as a predictor of driving accident involvement. Journal of Occupational and Organizational Psychology, 67, 173-182.

Braitman, K. A., Kirley, B. B. McCartt, A. T., and Chaudhary, N. K. (2008). Crashes of novice teenage drivers: Characteristics and contributing factors. Journal of Safety Research, $39,47-54$.

Carroll, C. L., and Waller, P. (1980). Analysis of fatal and non-fatal motorcycle crashes and comparisons with passenger cars. In Proceedings of the International Motorcycle Safety Conference. Maryland: Motorcycle Safety Foundation.

Chapman, P., Underwood, G., and Roberts, K. (2002). Visual search patterns in trained and untrained novice drivers. Transportation Research Part F, 5, 157-167.

Clarke, D.D., Ward, P.J., Bartle, C., and Truman, W.A. (2007) The role of motorcyclist and other driver behaviour in two types of serious accident in the UK. Accident Analysis and Prevention, 39, 5, 974-981.

Crundall, D. (2009). The Deceleration Detection Flicker Test: A measure of experience? Ergonomics, 52, 6, 674 - 684. 
Crundall, D. Andrews, B., van Loon, E., and Chapman, P. (2010). Commentary training improves responsiveness to hazards in a driving simulator. Accident Analysis and Prevention, $42,6,2117-2124$.

Crundall, D., Crundall, E., Clarke, D., and Shahar, A. (2012). Why do car drivers fail to give way to motorcycles at t-junctions? Accident Analysis and Prevention, 44,88-96.

Crundall, E. Crundall, D, and Stedmon, A. (2012). Negotiating left-hand and right-hand bends: A motorcycle simulator study to investigate experiential and behaviour differences across rider groups. PLoS ONE, 7, 1, e29978.

Crundall, E. Stedmon, A., Saikayasit, R., and Crundall, D, (2013). A Simulator Study Investigating How Motorcyclists Approach Side-road Hazards. Accident Analysis and Prevention, 51, 42-50.

Crundall, D. E. and Underwood, G. (1998).The effects of experience and processing demands on visual information acquisition in drivers. Ergonomics, 41, 4, 448-458.

Crundall, D., Underwood, G., and Chapman, P. (1999). Driving experience and the functional field of view. Perception, 28, 1075-1087.

Crundall, D., Underwood, G., and Chapman, P. (2002). Attending to the peripheral world while driving. Applied Cognitive Psychology, 16, 459-475.

Crundall, D., van Loon, E., Stedmon, A., and Crundall, E. (2013). Motorcycling experience and hazard perception. Accident Analysis and Prevention, 50, 456-464

Department for Transport (2012a). Road traffic (vehicle miles) by vehicle type in Great Britain; Table TRA0101. Accessed on Feb 6, 2013 from www.gov.uk/government/uploads/.../tra0101.xls 
Department for Transport (2012b). Reported Road Casualties Great Britain 2011.Accessed on Feb 6, 2013 from

www.gov.uk/government/uploads/system/uploads/attachment_data/file/9280/rrcgb2011complete.pdf

Divey, S. T. (1991). The accident liabilities of advanced drivers. Transportation Road Research Laboratory, Crowthorn.

Duncan, J., Williams, P., and Brown, I. (1991) Components of driving skill: experience does not mean expertise. Ergonomics, 34, 919-937

Elliot, M.A, Baughan, C.J., and Sexton, B.F. (2007) Errors and violations in relation to motorcyclists' crash risk. Accident Analysis and Prevention, 39, 491-499.

Fuller, R., McHugh, C., Pender, S., (2008). Task difficulty and risk in the determination of driver behaviour. Revue européenne de psychologie appliqée, 58, 13-21.

Gregersen, N. P. (1996). Young drivers' overestimation of their own skill - an experiment on the relation between training strategy and skill. Accident Analysis and Prevention, 28, 243250.

Groeger, J. A., and Banks, A. P. (2007). Anticipating the content and circumstances of skill transfer: Unrealistic expectations of driver training and graduated licensing? Ergonomics, 50, 1250-1263.

Hoinville, G., Berthould, R., Mackie, A.M., (1972). A study of accident rates amongst motorists who passed or failed an advanced driving test. Transport Road and Research Laboratory (TRRL) Report 499, Crowthorne. 
Horswill, M.S., and McKenna, F.P., (2004). Drivers' hazard perception ability: Situation awareness on the road. In S. Banbury and S. Tremblay (eds.) A Cognitive Approach to Situation Awareness. Aldershot, UK: Ashgate.

Horswill, M. S., Kemala, C. N., Wetton, M., Scialfa, C. T., and Pachana, N. A., (2010). Improving older drivers' hazard perception ability. Psychology and Aging, 25, 464-469.

Hosking, S.G., Liu, C.C., and Bayly, M. (2010) The visual search patterns and hazard responses of experienced and inexperienced motorcycle riders. Accident Analysis and Prevention, 42, 196-202.

Jackson, A. L., Chapman, P., and Crundall, D. (2009). What happens next? Predicting other road users' behaviour as a function of driving experience and processing time. Ergonomics, $52,2,154-164$.

Katila, A., Keskinen, Esko, Hatakka, M., and Laapotti, S. (2004). Does increased confidence among novice drivers imply a decrease in safety?: The effects of skid training on slippery road accidents. Accident Analysis and Prevention, 36, 543-550.

Koustanaï, A., Boloix, E., van Elslande, P., and Bastien, C., (2008). Formation of expectations while driving: Influence of the possibility and the necessity to anticipate on the ability to identify danger. Transportation Research Part F, 11, 147-157.

Liu, C.C., Hosking, S.G., and Lenné, M.G. (2009) Hazard perception abilities of experienced and novice motorcyclists: An interactive simulator experiment. Transportation Research Part $F, 12,325-334$ 
Lynham, D., Broughton, J., Minton, R. and Tunbridge, R. J. (2001). An Analysis of Police Reports of Fatal Accidents Involving Motorcycles. TRL Report No. 492. Crowthorne: Transport Research Laboratory.

M.A.G. UK, (2006). How close is too close? Concerning car collisions with motorcycles. www.network.mag-uk.org/smidsy/How\%20Close\%20is\%20Too\%20Close.pdf, (accessed on 06/04/06).

Mannering, F.L. and Grodsky, L. L. (1995). Statistical analysis of motorcyclists' perceived accident risk. Accident Analysis and Prevention, 27, 21-31.

Maycock, G. Lockwood, C. R. and Lester, J. F. (1991). The accident liability of car drivers. TRL Report 315. Crowthorne: Transport Research Laboratory.

Most, S. B., and Astur, R. S. (2007). Feature-based attentional set as a cause of traffic accidents. Visual Cognition, 15, 125-132

Pollatsek, A., Narayanaan, V., Pradhan, A., and Fisher, D. L., (2006). Using eye movements to evaluate a PC-based risk awareness and perception training program on a driving simulator. Human Factors, 48, 447-464.

Rosenbloom, T., Shahar, A., Elharar, A., and Danino, O., (2008). Risk perception of driving as a function of advanced training aimed at recognizing and handling risks in demanding driving situations. Accident Analysis and Prevention, 40, 697-703.

Shahar, A., Poulter, D., Clarke, D., and Crundall, D. (2010). Motorcyclists' and car drivers' responses to hazards. Transportation Research Part F, 13, 243-254. 
Stedmon, A. W., Crundall, D., Crundall, E., Irune, A., van Loon, E., Ward, P., and Greig, N., (2011). Investigating motorcycle rider behaviour: Developing an integrated experiment approach. Advances in Transportation Studies, special issue 2010, 63-78.

Stedmon, A. W., Crundall, D., Crundall, E., Saikayasit, R., van Loon, E., Irune, A., Ward, P., and Greig, N. (2011). 'STISIM-drive' meets 'MotorcycleSim': Using driving simulation software to develop a unique motorcycle simulator for rider behavior research. Communications in Computer and Information Science, 174, 76-80.

Summala, H., Pasanen, E., Räsänen, M., and Sievänen (1996). Bicycle accidents and drivers' visual search at left and right turns. Accident Analysis and Prevention, 28, 147-153.

Underwood, G., (2007) Visual attention and the transition from novice to advanced driver. Ergonomics, 50, 1235-1249

Underwood, G., Chapman, P., Brocklehurst, N., Underwood, J., and Crundall, D. (2003). Visual attention while driving: Sequences of eye fixations made by experienced and novice drivers. Ergonomics, 46, 629-646.

van Elsland, P., and Faucher-Alberton, L. (1996). When expectancies become certainties: A potential adverse effect of experience. In T. Rothengatter and E. Carbonell Vaya (Eds.) Traffic and Transport Psychology: Theory and Application. Oxford: Pergamon.

Verster, J. C., Taillard, J., Sagaspe, P., Olivier, B., and Philip, P. (2011). Prolonged nocturnal driving can be as dangerous as severe alcohol-impaired driving. Journal of Sleep Research, 20,585-588. 
Vidotto G, Bastianelli A, Spoto A, Sergeys F (2011) Enhancing hazard avoidance in teennovice riders. Accident Analysis and Prevention 43, 1, 247-252.

Wilde, G.J.S., (1982). The theory of RHT: implications for safety and health. Risk Analysis, 2, 209-226.

Wilde, G.J.S., (1988). Risk homeostasis theory and traffic accidents: propositions, deductions and discussion of dissension in recent reactions. Ergonomics, 3, 441-468. 


\section{TABLES}

Table 1. Rider demographics

\begin{tabular}{cccc}
\hline & Novice & Experienced & Advanced \\
\hline \hline Mean Age & 27 & 41 & 47 \\
$\begin{array}{c}\text { Mean Experience in } \\
\text { years }\end{array}$ & $1^{*}$ & 16 & 17 \\
$\begin{array}{c}\text { Mean Annual } \\
\text { Mileage }\end{array}$ & 3700 & 4300 & 7400 \\
$\begin{array}{c}\text { Hours riding per } \\
\text { week }\end{array}$ & 7.7 & 7.3 & 8.8 \\
\hline
\end{tabular}

*Includes time spent in training prior to passing their Compulsory Basic Training test

Table 2. Frequency of motorcycle crashes across rider groups

\begin{tabular}{cccc}
\hline & Novice & Experienced & Advanced \\
\hline \hline $\begin{array}{c}\text { Collisions with pre- } \\
\text { specified hazards }\end{array}$ & 12 & 13 & 5 \\
Other crashes & 3 & 1 & 5 \\
Total & 13 & 14 & 10 \\
\hline
\end{tabular}

Table 3. Cronbach's alpha for the five factors of the MRBQ

\begin{tabular}{ccccc}
\hline MRBQ factors & Cronbach's & \multicolumn{3}{c}{ Mean score } \\
\cline { 3 - 5 } & alpha & Novice & Experienced & Advanced \\
\hline \hline Traffic Errors & 0.74 & 1.96 & 1.88 & 1.62 \\
Speed Violations & 0.75 & 3.09 & 3.13 & 2.42 \\
Stunts & 0.77 & 1.48 & 1.69 & 1.29 \\
Safety Equipment & 0.50 & & & \\
Control Errors & 0.39 & & & \\
\hline
\end{tabular}




\section{FIGURE LEGENDS}

Figure 1. The average speed of the three rider groups in 60 and $40 \mathrm{mph}$ speed limit zones (left panel) and the average amount of time spent speeding in the 60 and $40 \mathrm{mph}$ zones (right panel). Standard error bars are included.

Figure 2. The mean position of riders in the lane (left panel; zero represents the centre line of the road, while a score of -12 would represent riding on the outermost edge of the road) and the mean variance in lateral position within the lane across the three driver groups for 60 and $40 \mathrm{mph}$ zones (with standard error bars).

Figure 3. The average maximum braking effort across the three driver groups for 60 and $40 \mathrm{mph}$ zones (with standard error bars).

Figure 4. The mean speed (left panel) and mean variance of lateral position (right panel) of the three rider groups across the four classifications of road geometry (with standard error bars). 
Figure 1.
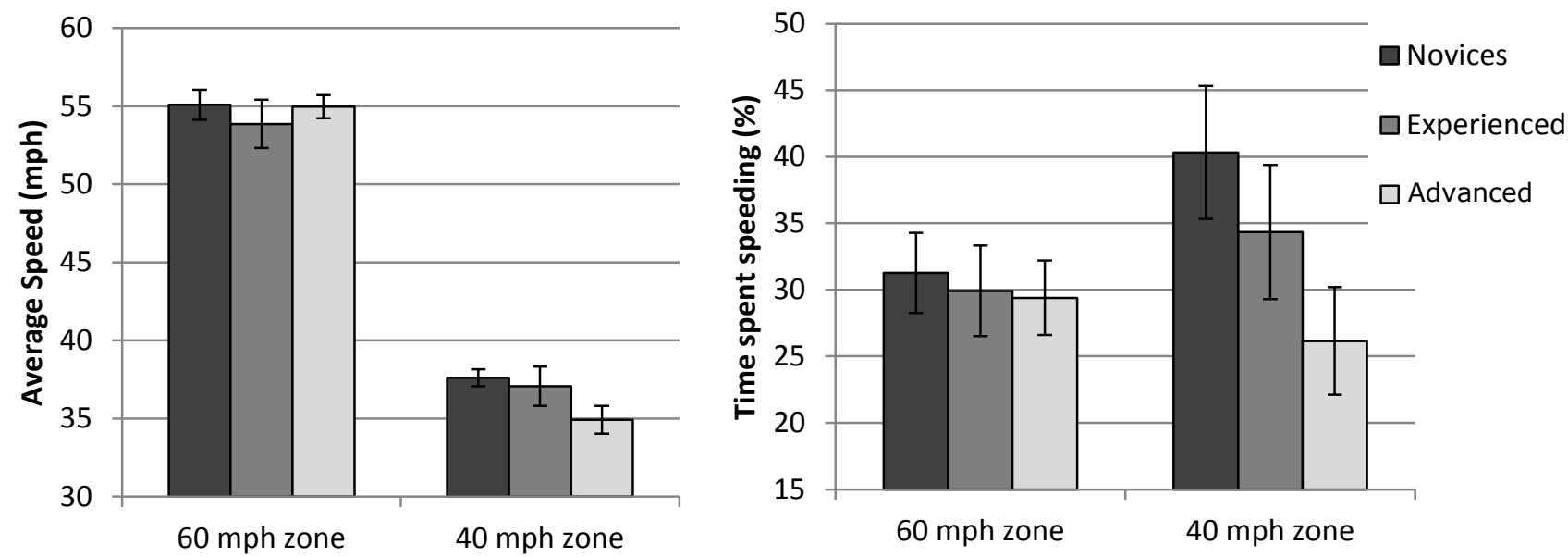
Figure 2.
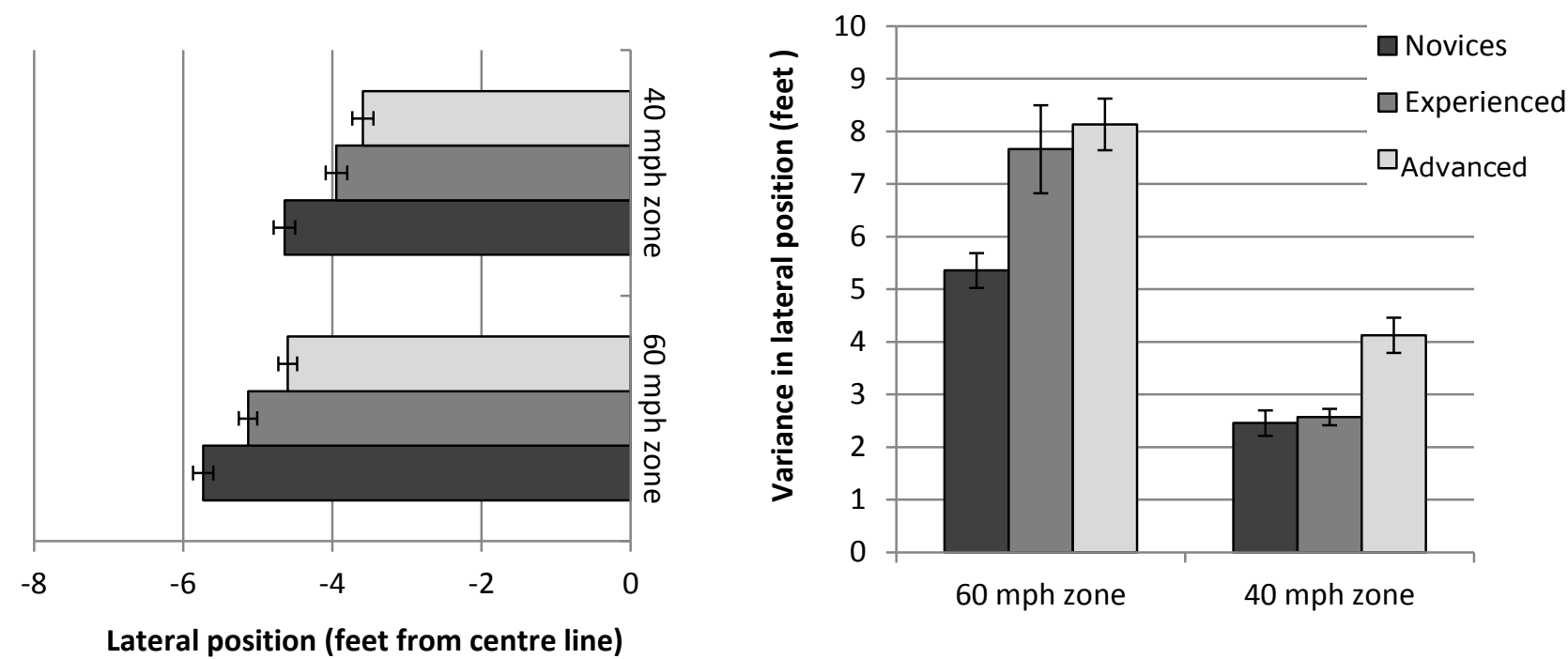
Figure 3.

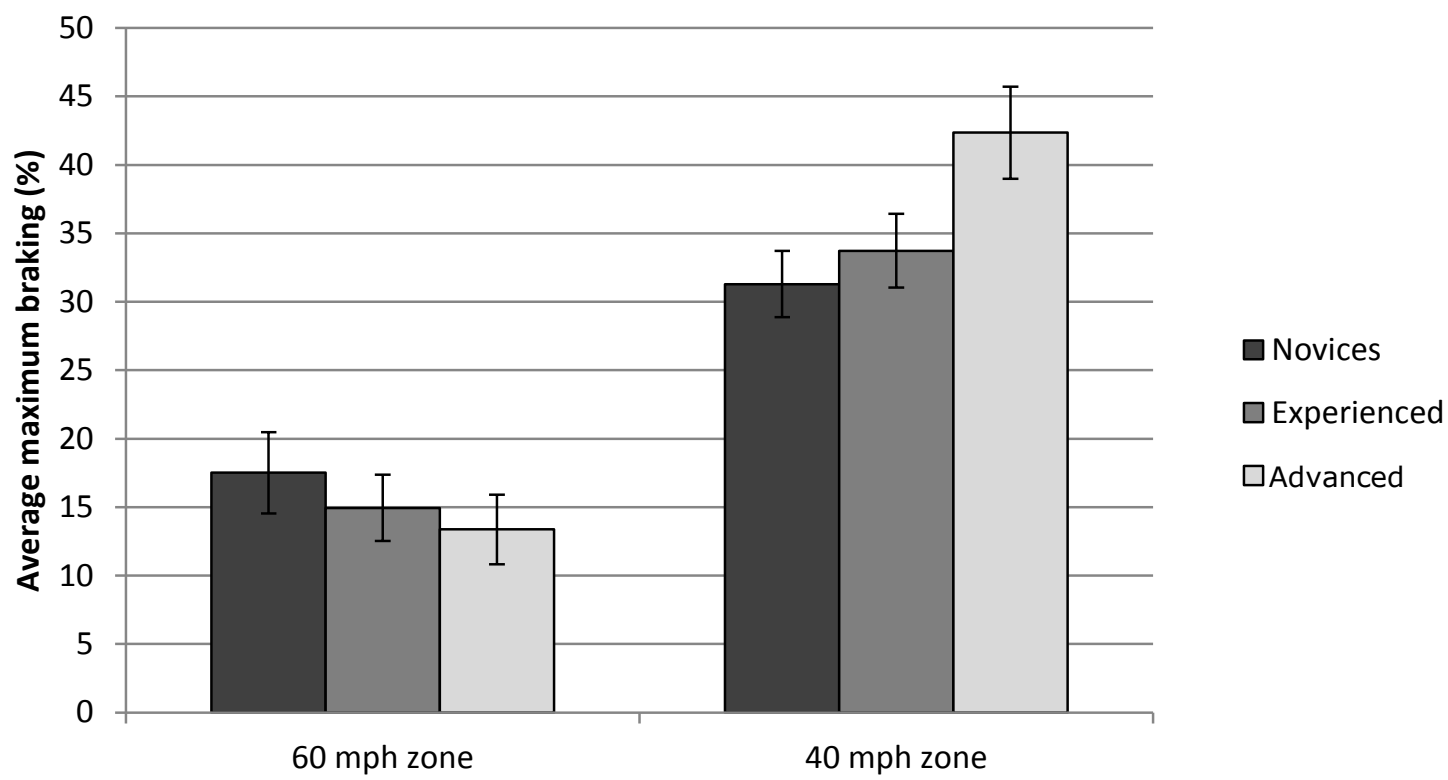


Figure 4.
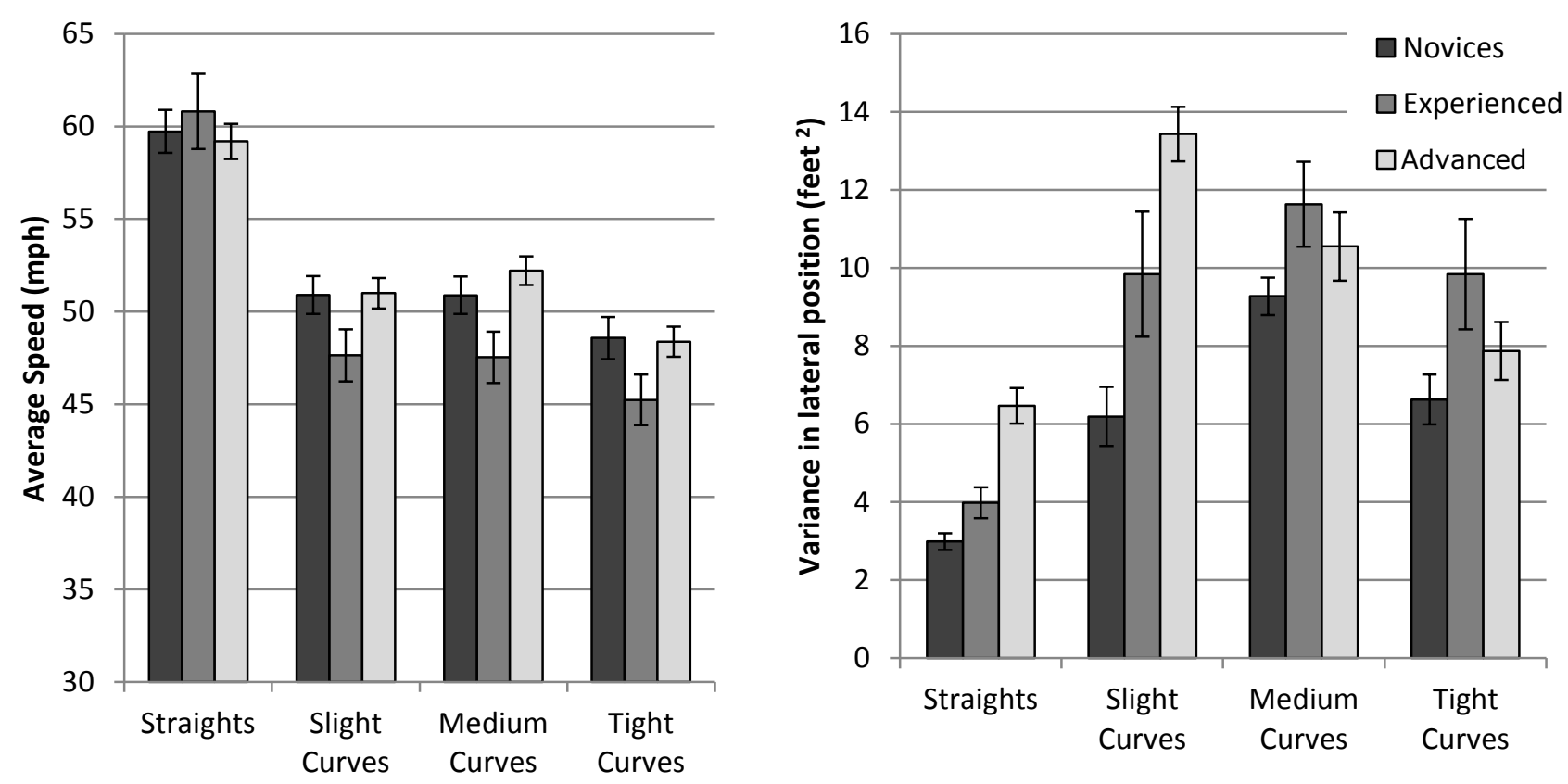